\title{
Chaos of Double Diffusive Convection in the Presence of Feedback Control Strategy
}

\author{
Zailan Siri ${ }^{1}$, Siti Norfarahin Hasan ${ }^{1}$, Rose Irnawaty Ibrahim ${ }^{2}$ \\ ${ }^{1}$ Institute of Mathematical Sciences, University of Malaya, 50603 Kuala Lumpur, Malaysia. \\ 2 Faculty of Science and Technology, Universiti Sains Islam Malaysia, 71800 Bandar Baru Nilai, Negeri \\ Sembilan, Malaysia. \\ * Corresponding author. Tel.: +603-79674335; email: zailansiri@um.edu.my \\ Manuscript submitted November 12, 2017; accepted January 29, 2018. \\ doi: 10.17706/ijapm.2018.8.2.10-17
}

\begin{abstract}
Chaos in double diffusive convection was investigated in this paper. A five-dimensional model of chaotic system was obtained using the Galerkin truncated approximation. The results showed, the transition from steady convection to chaos via a Hopf bifurcation produced a limit cycle which may be associated with a homoclinic explosion at a slightly subcritical value of the Rayleigh number.
\end{abstract}

Key words: Chaos, double-diffusive convection, concentration, limit cycle.

\section{Introduction}

Chaos theory is the concept of sensitive on initial conditions where small difference on initial condition may produce the large variations in the long term behavior of the system. This behavior also namely "butterfly effect" related to work done by [1] where it is already described by Henri Poincare in 1890 in the literature in a particular case of the three body problem. In dynamical system, chaos behavior has been studied intensively (see [2]-[5]) and in convection of fluid. In this study, we focused on the behavior of chaos in convection of fluid.

The investigation of free convection in Rayleigh-Bènard problem is receiving higher attention due to its wide applications in different disciplines such as astrophysics, oceanography, engineering and geology. A two-dimensional fluid cell heated from below and cooled from above (also known as the Rayleigh-Bènard problem) was studied by [1] (known as Lorenz's system) in order to model the unpredicted behavior in weather. He then came out with the three-dimensional set of partial differential equation known as model of fluid convection and suggested that difficulty in reaching good accuracy exists in a very long range forecasting because this model gives rise to chaotic behavior [6].

Researchers adopted the ideas of Lorenz's system [1] to other types of problems. In 1997, [7] modified the Lorenz's system by including two additional term: intensifies the vorticity and stabilizes the temperature field to investigate the convective motion heated from below with a sinusoidal differential heating. From the observation, they found that, the onset of chaotic convection is suppressed by the temperature difference and the marginal Rayleigh number is proportional to $h^{2}$, where $h$ is the horizontal difference in Rayleigh numbers. A chaotic mathematical model has been proposed by [6] to provide a profile of the chaotic attractor associated with the Rayleigh-Bènard problem in a plane fluid motion. The results showed the attractor of the Lorenz's system is a cross-section of the attractor of the proposed model. 
Chaos convection in fluid layer heated from below has been studied by [8] in order to obtain its subcritical transitions and hysteresis. Using the method of weak non-linear analysis theory and Adomian decomposition method, he shows the results is in a very good agreement for the transition from the steady solution to chaos in the neighbourhood of the convective solutions. His finding also explains the transition from steady convection to chaos is via a solitary limit cycle followed by homoclinic explosion at a subcritical value of Rayleigh number.

Research in double-diffusive convection begins after work done by sea-going oceanographers in order to measure the fluctuation of temperature and salinity as a function of depth as stated in the paper of [9]. Then, [10] and [11] studied the transition to chaos in double-diffusive convection with stress free boundary conditions where the oscillatory solution exists. They showed that the instability of fluid becomes oscillatory when thermal Rayleigh number is raised and the truncated model suggests that the appearance of chaos is associated with heteroclinic bifurcations.

Two-dimensional thermosolutal convection between free boundaries was studied numerically by [12] and Huppert and Moore (1976) as cited in [10]. From their observation, they found that when solutal Rayleigh number is large enough, the oscillations underwent a bifurcation to asymmetry as thermal Rayleigh number increased and for the larger values of solutal Rayleigh number, the transition from chaos to steady motion occurs. While the study of feedback control was found in [13-23].

The objective of the present paper is to study the weak turbulence and chaos for low Prandtl number in double-diffusive convection involving temperature and concentration as the thermal Rayleigh number increases with rigid, no-slip horizontal boundary condition in the presence of feedback control. Applying the truncated Galerkin approximation to the governing equations yields an autonomous system with five ordinary differential equations which can be used to understand low-dimensional dynamics before moving to studies more complex systems. Note that if the system in absence of feedback control, the system were reduced to the system solved by [24].

\section{Mathematical Formulation}

Consider a two-dimensional layer of fluid of depth $\mathrm{H}$ subject to gravity and heated from below as shown in Fig. 1, a Cartesian coordinate system is used such that the vertical axis $z$ is collinear with gravity, that is, $\hat{\mathbf{e}}_{\mathrm{g}}=\hat{\mathbf{e}}_{\mathrm{z}}$. A sensor array is placed at the top of the fluid layer. The bottom boundary consists of actuators. The bottom temperature is controlled as a function of the temperature at the top.

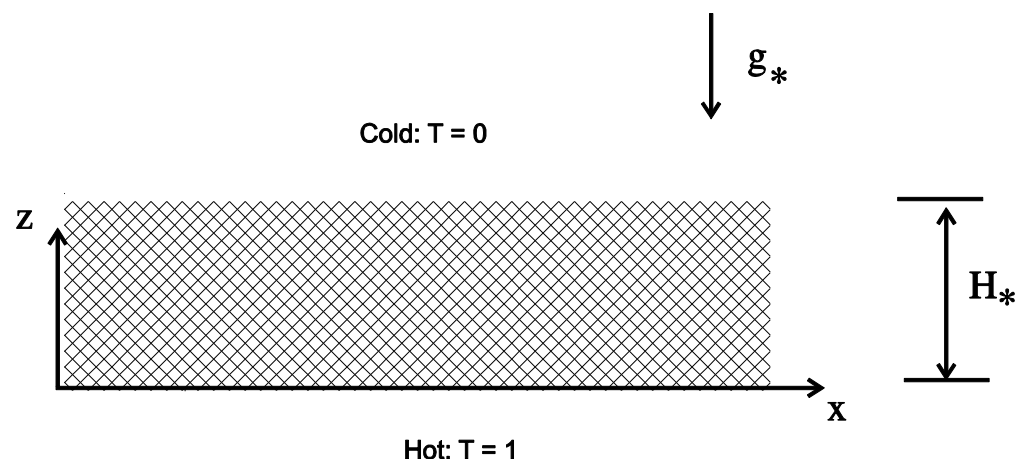

Fig. 1. Physical model.

The two long walls are maintained at temperature $T_{H}$ and $T_{C}$ while concentration is assumed linear and takes the form of $\rho=\rho_{0}\left[1-\alpha\left(T-T_{C}\right)+\alpha_{S}\left(S-S_{C}\right)\right]$, where $\alpha$ and $\alpha_{S}$ is volume expansion coefficient due to variations of thermal and solute concentration. The Boussinesq approximation is applied for the 
effects of density variations for the gravity term in momentum equation.

Therefore, the set of equations governing the conservation of mass, momentum, energy and concentration for fluid flow is given by,

$$
\begin{gathered}
\nabla \cdot \mathbf{V}=0 \\
\rho_{0}\left[\frac{\partial \mathbf{V}}{\partial t}+\mathbf{V} \cdot \mathbf{V}\right]=-\nabla p+\nabla^{2} \mathbf{V}+\rho \hat{\mathbf{e}}_{\mathbf{z}} \\
\frac{\partial T}{\partial t}+\mathbf{V} \cdot \nabla T=\eta \nabla^{2} T \\
\frac{\partial S}{\partial t}+\mathbf{V} \cdot \nabla S=\eta \nabla^{2} S
\end{gathered}
$$

Introduce the following transformation,

$$
\begin{aligned}
& V_{*}=\frac{H_{*}}{\eta_{*}} V, \quad p_{*}=\frac{H_{*}^{2}}{\rho_{0} \eta_{*}^{2}} p, \quad T_{*}=\frac{T-T_{C}}{\Delta T_{C}}, \\
& S_{*}=\frac{S-S_{C}}{\Delta S_{C}}, \quad\left(x_{*}, y_{*}, z_{*}\right)=H_{*}(x, y, z), \quad t_{*}=\frac{t H_{*}^{2}}{\eta_{*}},
\end{aligned}
$$

where $\mathbf{V}_{*}=\left(u_{*}, v_{*}, w_{*}\right)$ is the velocity component, $p_{*}$ is the pressure, $T-T_{C}$ and $S-S_{C}$ is the temperature and solute concentration variations, $\eta_{*}$ is the effective thermal diffusivity, $v_{*}$ is fluid's viscosity.

In this model, all the boundaries are rigid and the solution must follow the impermeability conditions there, that is $\mathbf{V}_{*} \cdot \hat{\mathbf{e}}_{n}=0$ on the boundaries, where $\hat{\mathbf{e}}_{n}$ is a unit vector normal to the boundary. The temperature and solute concentration boundary conditions are $T=S=1$ at $z=0$, and $T=S=0$ at $z=1$.

For convective rolls having axes parallel to the shorter dimension (i.e $y=0$, ) $v=0$, apply the curl operator on eq. (2) to eliminate the pressure and introducing the stream function defined by $u=\frac{\partial \psi}{\partial z}$ and $w=-\frac{\partial \psi}{\partial x}$, we get,

$$
\begin{gathered}
{\left[\frac{1}{\operatorname{Pr}}\left(\frac{\partial}{\partial t}+\frac{\partial \psi}{\partial z} \frac{\partial}{\partial x}-\frac{\partial \psi}{\partial x} \frac{\partial}{\partial z}\right)-\nabla^{2}\right] \nabla^{2} \psi=\operatorname{Ra} \frac{\partial T}{\partial x}+\mathrm{R}_{S} \frac{\partial S}{\partial x}} \\
\frac{\partial T}{\partial t}+\frac{\partial \psi}{\partial z} \frac{\partial T}{\partial x}-\frac{\partial \psi}{\partial x} \frac{\partial T}{\partial z}=\frac{\partial^{2} T}{\partial x^{2}}+\frac{\partial^{2} T}{\partial z^{2}} \\
\frac{\partial S}{\partial t}+\frac{\partial \psi}{\partial z} \frac{\partial S}{\partial x}-\frac{\partial \psi}{\partial x} \frac{\partial S}{\partial z}=\frac{1}{\mathrm{Le}}\left(\frac{\partial^{2} T}{\partial x^{2}}+\frac{\partial^{2} T}{\partial z^{2}}\right)
\end{gathered}
$$

where, 


$$
\operatorname{Pr}=\frac{v_{*}}{\eta_{*}}, \mathrm{Ra}=\frac{\alpha_{*} \Delta T_{C} g_{*} H_{*}^{3}}{\eta_{*} v_{*}}, \mathrm{R}_{S}=\frac{\alpha_{S^{*}} \Delta S_{C} g_{*} H_{*}^{3}}{\eta_{*} \nu_{*}}, \mathrm{Le}=\frac{\eta_{*}}{\eta_{S^{*}}}
$$

which are, respectively, the Prandtl number, the Rayleigh number, the solutal Rayleigh number and the Lewis number. The boundary conditions for the stream function are $\psi=0$ on the horizontal boundaries. Eqs. (6)-(8) form a nonlinear coupled system which together with the corresponding boundary conditions allow for a basic motionless conduction solution.

\section{Dimished Set of Equations}

In order to obtain the solution to Eqs. (6)-(8), we represent the stream function and temperature distribution in the form

$$
\begin{gathered}
\psi=A_{11} \sin (\kappa x) \sin (\pi x), \\
T=\frac{1}{2}\left[1-K-(2-K) z+B_{11} \cos (\kappa x) \sin (\pi x)+B_{02} \sin (2 \pi z)\right], \\
S=1-z+C_{11} \cos (\kappa x) \sin (\pi x)+C_{02} \sin (2 \pi z),
\end{gathered}
$$

where $K$ is the scalar gain controller as discussed in [13] and [14].

Substituting eqs. (10)-(12) into eqs. (6)-(8), multiplying the equations by the orthogonal eigenfunctions corresponding to eqs. (10)-(12), and then integrating them over the spatial domain yields a set of five ordinary differential equations for the time evolution of the amplitudes,

$$
\begin{gathered}
\frac{\mathrm{d}}{\mathrm{d} \tau} \bar{A}_{11}=-\operatorname{Pr}\left(\bar{B}_{11}+\bar{A}_{11}-\bar{C}_{11}\right), \\
\frac{\mathrm{d}}{\mathrm{d} \tau} \bar{B}_{11}=-\bar{B}_{11}+\mathrm{R} \bar{A}_{11}(K-2)-\bar{A}_{11} \bar{B}_{02}, \\
\frac{\mathrm{d}}{\mathrm{d} \tau} \bar{B}_{02}=\bar{A}_{11} \bar{B}_{11}-\lambda \bar{B}_{02}, \\
\frac{\mathrm{d}}{\mathrm{d} \tau} \bar{C}_{11}=-\frac{\bar{C}_{11}}{\mathrm{Le}}-\mathrm{R}_{S} \bar{A}_{11}-\bar{A}_{11} \bar{C}_{02}, \\
\frac{\mathrm{d}}{\mathrm{d} \tau} \bar{C}_{02}=\bar{A}_{11} \bar{C}_{11}-\frac{\lambda}{\mathrm{Le}} \bar{C}_{02} .
\end{gathered}
$$

In eqs. (13)-(17), the time, the amplitudes, the Rayleigh number and the solutal Rayleigh number were rescaled and the following notation was introduced,

$$
\begin{aligned}
& \bar{A}_{11}=\frac{\kappa / \kappa_{c r}}{\left(\kappa / \kappa_{c r}\right)^{2}+2} A_{11}, \quad \bar{B}_{11}=\kappa_{c r} \mathrm{R} B_{11}, \bar{B}_{02}=\pi \mathrm{R} A_{02}, \quad \bar{C}_{11}=\kappa_{c r} \mathrm{R}_{S} C_{11}, \bar{C}_{02}=\pi \mathrm{RC}_{02}, \\
& \mathrm{R}=\frac{\mathrm{Ra}}{\mathrm{Ra}_{c}}, \quad \mathrm{R}_{S}=\frac{\mathrm{Rs}}{\mathrm{Rs}_{c}}, \quad \tau=\left(\kappa^{2}+\pi^{2}\right) t, \lambda=\frac{8}{\left(\kappa / \kappa_{c r}\right)^{2}+2}, \mathrm{Ra}_{c}=\mathrm{Rs}_{c}=\frac{\left(\kappa^{2}+\pi^{2}\right)^{3}}{\kappa^{2}}, \kappa_{c r}=\frac{\pi}{\sqrt{2}} .
\end{aligned}
$$




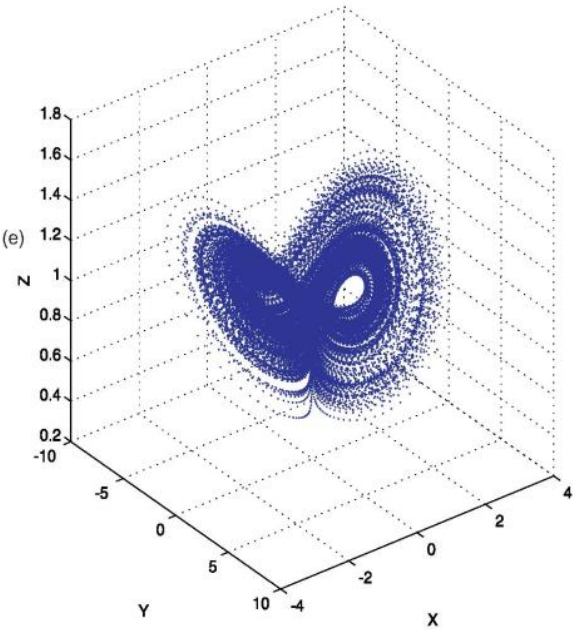

(a)

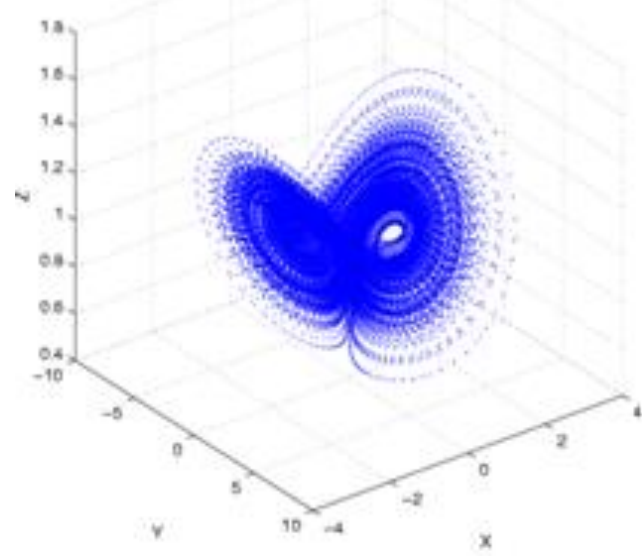

(c)

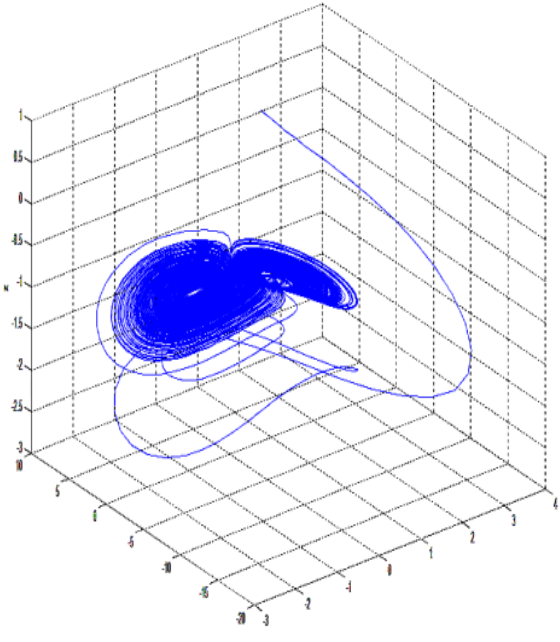

(b)

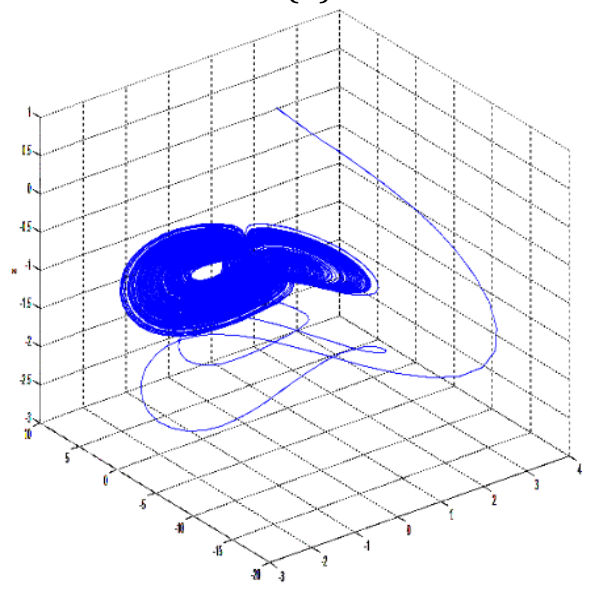

(d)

Fig. 2. Phase portraits for the case $L e=0.1, K=0$ (left), $K=1$ (right) $\quad$ (a), $R=300, R s=15$ (b) $R=200$, $R s$ $=15$ (b) $R=400, R s=20$ and (d) $R=230, R s=30$.

Rescaling the equation again in the form of,

$$
X=\frac{\bar{A}_{11}}{\sqrt{\lambda(\mathrm{R}-1)}}, \quad Y=\frac{\bar{B}_{11}}{\sqrt{\lambda(\mathrm{R}-1)}}, \quad \mathrm{Z}=\frac{\bar{B}_{02}}{\mathrm{R}-1}, \quad U=\frac{\bar{C}_{11}}{\sqrt{\lambda(\mathrm{R}-1)}}, \quad W=\frac{\bar{C}_{02}}{\mathrm{R}-1},
$$

to provide the following set of scaled equations which are equivalent to Eqs. (13)-(17):

$$
\begin{gathered}
\dot{X}=-\operatorname{Pr}(Y+X-U), \\
\dot{Y}=\mathrm{R} X(K-2)-Y-(\mathrm{R}-1) X Z, \\
\dot{Z}=\lambda(X Y-Z), \\
\dot{U}=-\mathrm{R}_{S} X-\frac{U}{\mathrm{Le}}-(\mathrm{R}-1) X W,
\end{gathered}
$$




$$
\dot{W}=-\lambda\left(X Z+\frac{W}{\mathrm{Le}}\right)
$$

where the dots denote time derivatives $\frac{\mathrm{d}}{\mathrm{d} \tau}()$. Note that if $K=0$ the problem is reduced to the study of [24].

\section{Results and Discussion}

In this paper, we investigate the chaos behaviour with low Prandtl number in double-diffusive convection in the present of feedback control strategy. We obtained a system (18) - (22) that provides a set of non-linear equations with five parameters. Parameter Pr is a Prandtl number, $\mathrm{R}$ is a Rayleigh number, Rs is a solutal Rayleigh number and Le is a Lewis number. The value of $\lambda$ has to be consistent with the wave number at the convection threshold, a requirement for the convection cells to fit into the domain and fulfill the boundary conditions.

The Lorenz-like equations have been extensively analyzed and solved for parameter values corresponding to convection in pure fluids and even there, the parameter values most regularly used correspond to $\operatorname{Pr}=10$ and $\lambda=8 / 3$ which is consistent with the the study of [24]. All solutions were obtained using the same initial conditions, which were selected at $\tau=0: \mathrm{X}, \mathrm{Y}, \mathrm{Z}, \mathrm{U}, \mathrm{W}=0.9$. All computations were carried out with the value of maximum time, $\tau_{\max }=210$ and a step size $h=0.001$ running using built-in ODE45 method on MATLAB R2010a, which provides a numerical solution.

The phase potrait illustrated in Fig. 2 identified where the system chaotic. When we fixed Le $=0.1$ and increase Rs from 15 to 30 , shows the dynamical behaviour return to chaotic at $\mathrm{R}=300, \mathrm{~K}=0$ and $\mathrm{R}=200, \mathrm{~K}$ $=1$ respectively as shown in Fig. 2(a) and (b). It can be say that with the present of controller gain parameter it decrease the value of $\mathrm{R}$ where the chaotic bahaviour occur. The same phenomena also has been observe if we increase Rs (see Fig. 2 (c) and (d)).

\section{Conclusion}

In this work, we used ODE45 method to investigate the chaos for low Prandtl number in double-diffusive convection in fluid layer in the present of controller gain parameter. It is shown that the system become chaos if we at a lower $\mathrm{R}$ with the present of feedback control.

\section{Acknowledgment}

The authors acknowledge the financial support from University of Malaya, Grant No.: RG292-14AFR.

\section{References}

[1] Lorenz, E. N. (1963). Deterministic nonperiodic flow. Journal of the Atmospheric Sciences, 20, 130-141.

[2] Qi, G., Du, S., Chen, G., Chen, Z., \& Yuan, Z. (2005). On a four-dimensional chaotic system. Chaos, Solitons and Fractals, 23, 1671-1682.

[3] Chen, G., \& Ueta, T. (1999). Yet another chaotic attractor. International Journal of Bifurcation and Chaos, 9, 1465-1466.

[4] Liu, W. B., \& Chen, G. A. (2003). A new chaotic system and its generation. International Journal of Bifurcation and Chaos, 13, 261-267.

[5] L"u, J. H., Chen, G., \& Celikovsk'y, S. (2002). Bridge the gap between the lorenz system and the chen system. International Journal of Bifurcation and Chaos, 12, 2917-2926.

[6] Chen, Z. M., \& Price, W. G. (2006). On relation between Rayleigh-B'enard convection and Lorenz system. 
Chaos, Solitons and Fractals, 28, 571-578.

[7] Ookouchi, Y., \& Hada, T. (1997). Chaotic convection in a simple system modified by differential heating. Journal of the Physical Society of Japan, 66, 369-378.

[8] Vadasz, P. (2000). Subcritical transitions to chaos and hysteresis in a fluid layer heated from below. International Journal of Heat and Mass Transfer, 43, 705-724.

[9] Huppert, H. E., \& Turner, J. S. (1981). Double-diffusive convection. Journal of Fluid Mechanics, 106, 299-329.

[10] Knobloch, E., Moore, D. R., Toomre, J., \& Weiss, N. O. (1986). Transitions to chaos in two-dimensional double-diffusive convection. Journal of Fluid Mechanics, 166, 409-448.

[11] Bhattacharjee, J. K. (1987). Convection and chaos in fluids. World Scientific, Singapore.

[12] Veronis, G. (1968). Effect of a stabilizing gradient of solute on thermal convection. Journal of Fluid Mechanics, 34, 315-336.

[13] Roslan, R., Mahmud, M. N., Hashim, I. (2011). Effects of feedback control on chaotic convection in fluid-saturated porous media. International Journal of Heat and Mass Transfer, 54, 404-412.

[14] Mahmud, M. N., Hashim, I. (2011). Small and moderate Vadasz number chaotic convection in porous media in the presence of non-Boussinesq effects and feedback control. Physics Letters A, 375, 2382-2393.

[15] Hashim, I., \& Siri, Z. (2008). Stabilization of steady and oscillatory Marangoni instability in rotating fluid layer by feedback control strategy. Numerical Heat Transfer Part A, 54(6), 647-663.

[16] Siri, Z., \& Hashim, I. (2008). Control of oscillatory Marangoni convection in a rotating fluid layer. International Communications in Heat and Mass Transfer, 35(9), 1130-1133.

[17] Siri, Z., Mustafa, Z., \& Hashim, I. (2009). Effects of rotation and feedback control on Benard-Marangoni convection. International Journal of Heat and Mass Transfer, 52(25-26), 5770-5775.

[18] Hashim, I., \& Siri, Z. (2009). Feedback Control of Thermocapillary Convection in a Rotating Fluid Layer with Free-Slip Bottom. Sains Malaysiana, 38(1), 119-124.

[19] Siri, Z., \& Hashim, I. (2009). Control of Oscillatory of benard-marangoni convection in rotating fluid layer. Proceedings of International Conference on Signal Processing Systems (pp. 938-941).

[20] Shang, Y. (2012). Optimal attack strategies in a dynamic botnet defense model. Applied Mathematics and Information Sciences, 6(1), 29-33.

[21] Shang, Y. (2013). Optimal Control strategies for virus spreading in inhomogeneous epidemic dynamics. Canadian Mathematical Bulletin, 56(3), 621-629.

[22] Shang, Y. (2014). Continuous-time average consensus under dynamically changing topologies and multiple time-varying delays. Applied Mathematics and Computation, 244, 457-466.

[23] Shang, Y. (2014). Group consensus in generic linear multi-agent systems with inter-group non-identical inputs. Cogent Engineering, 1, 947761.

[24] Idris, R., Siri, Z., Hashim, I. (2013). On a Five-dimensional chaotic system arising from double-diffusive convection in a fluid layer. Abstract and Applied Analysis, 428327.

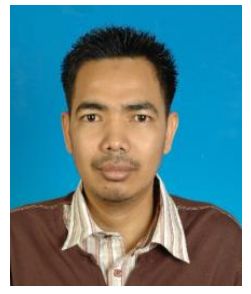

Zailan Siri was born in Melaka, Malaysia, in 1977. He received the B.Sc. in computational and industrial mathematics from the University of Malaya, Malaysia, in 2001, and the M.Sc. in numerical analysis from the Universiti Putra Malaysia, in 2004. Then, he received Ph.D. in mathematics from Universiti Kebangsaan Malaysia in 2012.

In 2002, he joined the Institute of Mathematical Sciences, University of Malaya, as a tutor, and in 2004 became a lecturer. Since July 2012, he has been with the Institute of Mathematical Sciences, University of Malaya, where he was a senior lecturer. His current research interests 
include numerical analysis, fluids flow problems, chaotic convection, and mortality study and pension scheme.

Dr. Siri was a memberships of Malaysian Mathematical Sciences Society and Golden Key Society Malaysia for life.

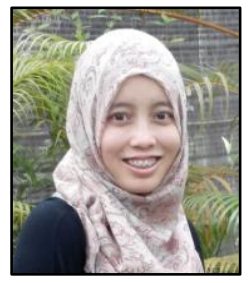

Siti Norfarahin Hasan was born in Kuala Lumpur, Malaysia, in 1986. She received the B.Sc. in mathematics from the University of Malaya, Malaysia, in 2010, and now she is M.Sc. in mathematics candidate at University of Malaya, Malaysia, in 2014.

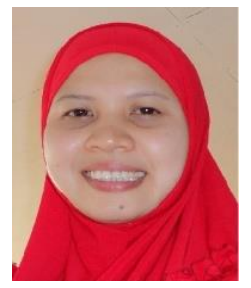

Rose Irnawaty Ibrahim was born in Kuala Lumpur, Malaysia, in 1976. She received the B.Sc. in computational and industrial mathematics from the University of Malaya, Malaysia, in 2000, and the M.Sc. in actuarial science from the Heriot-Watt University, U.K. in 2001. Then, she received Ph.D. in actuarial science from Universiti Teknologi Mara, Malaysia, in 2009.

In 2001, she joined the Institute of Mathematical Sciences, University of Malaya, as a tutor, and in 2006 became a lecturer. In February 2011, she joined the Faculty of Sciences and Technology, Universiti Sains Islam Malaysia as a senior lecturer. Her current research interests include mortality study and pension scheme, survival analysis, fuzzy and computational mathematics.

Dr. Ibrahim was a memberships of Malaysia Institute of Statictics in 2007 until 2008 and a memberships of Malaysian Mathematical Sciences Society and Golden Key Society Malaysia for life. 